Copyright (C) 2008 IEEE. Reprinted from Bioinformatics and Biomedical Engineering, 2008. ICBBE 2008. The $2^{\text {nd }}$ Internationa Conference on May 2008

This material is posted here with permission of the IEEE. Internal or personal use of this material is permitted. However, permission to reprint/republish this material for advertising or promotional purposes or for creating new collective works for resale or redistribution must be obtained from the IEEE by writing to pubs-permissions@ieee.org. By choosing to view this document, you agree to all provisions of the copyright laws protecting it. 


\title{
A New 3D Model-based Minimal Path Segmentation method for Kidney MR Images
}

\author{
$\mathrm{Ke} \mathrm{Li}^{1}$ and Baowei Fei ${ }^{1,2}$ \\ ${ }^{1}$ Departments of Radiology and ${ }^{2}$ Biomedical Engineering \\ Case Western Reserve University, Cleveland, OH, 44106
}

\begin{abstract}
We present a robust, automated, model-based segmentation method for kidney MR Images. We used dynamic programming and a minimal path approach to detect the optimal path within a weighted graph between two end points. We used an energy function to combine distance and gradient information to guide the marching curve and thus evaluate the best path and span a broken edge. We developed an algorithm to automate the placement of initial end points. Dynamic programming was used to automatically optimize and update end points in the procedure for searching curves. A deformable 3D model was generated using principle component analysis (PCA) and it was used as the prior knowledge for the selection of initial end points and for the evaluation of the best path. We used our minimal path method with surface models to segment mouse kidneys slice-by-slice. The method has been tested for kidney MR images of 44 mice. To quantitatively assess the automatic segmentation method, we compared the automatic segmentation results with manual segmentation. The average and standard deviation of the overlap ratios is $0.93 \pm 0.05$. The distance error between the automatic and manual segmentation is $0.85 \pm 0.41$ pixel. The $3 D$ automatic minimal path segmentation method is fast, accurate, and robust. It provides a useful tool for quantification and characterization of kidney MR images.
\end{abstract}

Keywords-3D; Segmentation; minimal path; deformable model; Dynamic Programming; polycystic kidney disease; MRI

\section{INTRODUCTION}

Autosomal-dominant polycystic kidney disease (ADPKD) is a common genetic disorder characterized by the formation of fluid-filled renal cysts that can eventually lead to renal failure. Serial MRI studies can provide high-resolution anatomic structure of the kidneys and thus could be a useful tool for the assessment of various therapies [1]. In this study, we develop a new 3D segmentation method to segment and measure the kidney volumes of transgenic mice with polycystic kidney disease (PKD) from MR images.

The organization of the paper is as follows. In section II, we discuss the details of proposed method. In section III, the method is applied to segment and measure the kidney MR images of transgenic mice.. The summary and conclusion are presented in section IV.

\section{SEGMENTATION METHODS}

\section{A. Energy Functions}

In a Riemannian space, the traditional energy function of snake [2] is transformed into a new function [3] as follows: $\min \int_{\Omega} \mathrm{g}(|\nabla \mathrm{I}(\mathrm{C}(v))|)^{2} \cdot\left|\mathrm{C}^{\prime}(v)\right| \mathrm{d} v$

where the Euclidean length of the contour $\mathrm{C}$ is given by $L(C)=\int_{\Omega}\left|C^{\prime}(v)\right| d \nu=\int_{\Omega} d s$. Therefore, the problem of image segmentation is transformed into a search for the global minimal path. This method has low computational complexity in high-order gradients and does not involve minimizing the corresponding Euler-Lagrange equation.

We incorporated distance and gradient information into an energy function to guide the marching curve toward the best path and span the broken edge. Our energy function is as follows:

$E(C)=\int_{\Omega}\left(\alpha E_{\text {model }}+\beta E_{\text {image }}+\gamma E_{\text {int }}\right) d v$

Where $\alpha, \beta$ and $\gamma$ are real positive weighting constants which balance the forces, $\Omega$ denotes the current curve body, $E_{\text {model }}$ can be calculated using the Bayes' Rule and the maximum posteriori probability (MAP) function,

$$
\begin{aligned}
& \Psi_{\mathrm{MAP}}=\operatorname{argmin}_{\Psi}\left\{v_{1} \int_{\mathrm{C}}|\Psi(\mathrm{C}(\mathrm{s}))| d s+v_{2} \int_{\Psi=0} \mathrm{G}(\mathrm{x}, \mathrm{y}) d x d y+\right. \\
& \left.\frac{1}{2} \alpha^{T} \sum_{k}^{-1} \alpha\right\}
\end{aligned}
$$

where $\Psi$ denotes the weighted graph that contains the estimated curve of the object, $\mathrm{G}(\mathrm{x}, \mathrm{y})$ denotes the weighted graph of the image. The first term represents the degree of the current curve matching the estimated distance map. The second term represents the degree of the estimated distance map matching the former distance map. The third term represents the probability of the estimated shape which is described later.

$$
E_{\text {image }}=\lambda \int_{C}\left|C_{S}\right| \mathrm{ds}-(1-\lambda) \int_{C} \mathrm{~g}(|\nabla \mathrm{I}(\mathrm{C}(v))|)^{2} d v
$$

where the first term denotes the degree of the curve $C$ in the distance map, which is the Euclidean length of the curve $C$; the second item denotes the gradient of the original image, which is used to regulate the searching when the distance map is not enough for guiding the curve in case of noises and lack of a real edge. $\lambda$ and $(1-\lambda)$ denote the weights of the distance and gradient information.

In the discrete case, $E_{\text {int }}=\left\|P_{i-1}-2 P_{i}+P_{i+1}\right\|$, is a smooth term.

\section{B. Kidney Model}

In our method, a deformable model for selecting initial points and evaluating the best path is created. For training images, we computed and obtained a kidney shape model using 
the principal component analysis (PCA) $[3,4]$. Using singular value decomposition (SVD), the matrix is decomposed as $P=U \sum V^{T}$. Matrix $\mathrm{U}$ is the model with orthogonal column vectors that consist of the modes of shape variation and diagonal matrix $\Sigma$ is composed of corresponding singular values. Given coefficient $\alpha$ and shape matrix $U_{k}$, we can estimate a novel shape $\eta$,

$\eta=U_{k} \alpha+\mu$

Which is represented by $\mathrm{k}$ principal components in a $\mathrm{k}$ dimensional vector of shape parameters $\alpha$ (where $k<n$ ). We assumed the shape parameter $\alpha$ was satisfied a Gaussian distribution as represented below:

$P(\alpha)=\frac{1}{\sqrt{(2 \pi)^{k}\left|\Sigma_{k}\right|}} \exp \left(-\frac{1}{2} \alpha^{T} \sum_{k}^{-1} \alpha\right)$

\section{Improved Dynamic Programming}

In our method, the images are transformed into a graphbased map. The graph-based search methods consider an image as a graph with a rectangular grid in which each pixel is a node. Boundary definition via dynamic programming can be formulated as a graph searching problem where the goal is to find the optimal path between a set of start nodes and a set of end nodes $[5,6]$.

The optimal path is defined as the minimum cumulative cost path, where the cumulative cost of a path is the sum of the local costs of the pixels on the path. To overcome the difficulty of searching the initial direction using dynamic programming, we developed an algorithm to incorporate eight directions into one universal searching map. As in Figure 1, we define a fixed direction window, any direction searching window map must be transformed into this fixed direction searching window. We defined the center point of the bottom line in this window as the start stage of dynamic programming, and defined each point of the last line as the end stage. Then, we can obtain several searched results and then evaluate and get the best one using previous energy function.

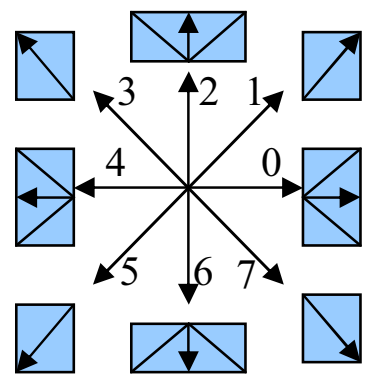

(a)

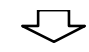

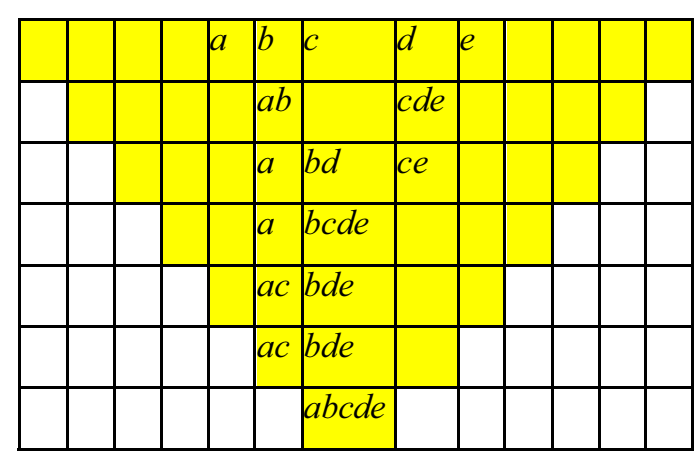

(b)

Figure 1. (a) Eight directions for searching windows. (b) Universal searching window. The center point of the bottom line represents the start stage, and the top line represents the end stage. The a, b, c, d, e represent 5 paths. A best minimal path will be selected by the algorithm among these paths. For example, Path $\mathrm{c}$ is the best one.

\section{EXPERIMENTS AND RESULTS}

We acquired kidney MR images from 44 mice using a Siemens Sonata $1.5 \mathrm{~T}$ scanner. We randomly selected 20 dataset for model training, and used the other 24 datasets to test the segmentation method.

For the training images, we first registered and manually segmented the 20 kidney dataset on each image. We then performed distance transformation under a fixed window size. After computing the mean of the training distance maps, we got a mean surface, $\mu=\frac{1}{n} \sum \mu$. Then, the variable $\mu$ is subtracted from each $\mu_{i}$ to create the mean-offset array, $\widehat{\mu}_{l}$, which is placed as a column vector in an $N^{d} \times n$ dimensional matrix $\mathrm{M}$. After using the formulas (5) and (6), we obtained the mean shape surface and singular value and orthogonal.

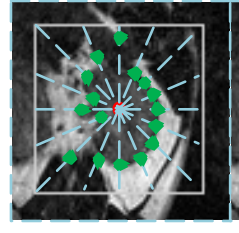

(a)

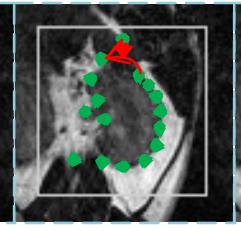

(b)

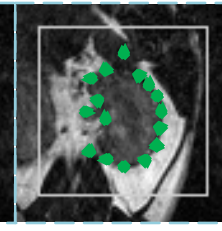

(c)

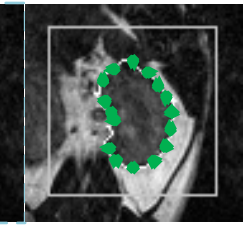

(d)
Figure 2. Procedure for selecting and updating end points. (a) Initial end points. Radial rays were generated from the window center of the distance map. If the weighting value of a point along the ray is zero, it is marked as an end point. If not, select the intersection point between the ray and the mean contour of the model as the marked end point. (b) Update end points. If there is a broken edge and the end point is far away from the real edge, the algorithm will use the model. The end point will be corrected towards the real edge (Arrow) after iterations. (c)-(d) End Points after one and two iterations.

During segmentation, we put the segmented object volumes into the box which sets the start slice and end slice. The object volume is between the two slices. The segmentation procedure was performed slice by slice. Each slice was segmented using our minimal path segmentation method with models. The slice 
images were first preprocessed by Canny edge detection and then by distance transformation. Figure 2 shows the procedure of creating and updating serial end points for minimal path searching. We placed the goal object located at the center of our searching window. Then, we selected the window center as the central point to create radius rays with an interval of a fixed angle (22.5 degrees in our case). So, an alignment of marked end points was generated.

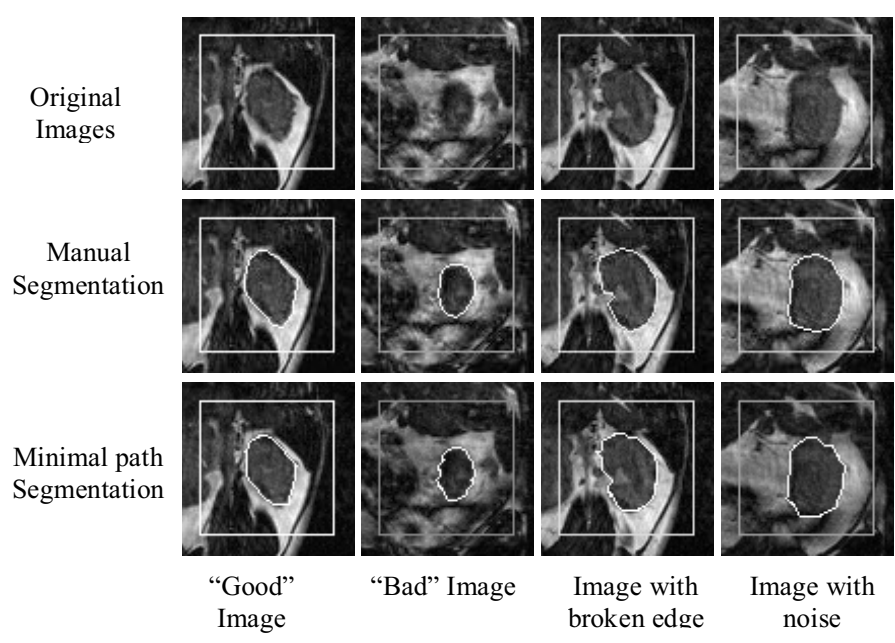

Figure 3 Automatic segmentation results for images with different quality. The method works well for both "good" and "bad" images. Image (a) is a typical kidney MR image of a mouse. The boundaries of the kidney on the images (b), (c) and (d) are not continuous. The automatic segmentation method works well for all the images.

At the beginning of searching the minimal path, we selected two interval points of the alignment and several subsequent neighbor points as the start and end stages for dynamic programming. After searching, several paths were generated. We used function (2) to evaluate and select the minimal energy path. Then, the middle point of this minimal path was selected as the next starting end point, rather than the next point of the original alignment. From this new starting point to its interval point, a new searching procedure was started again. Thus, the marked end points were continuously updated and optimized towards the direction of the real edge. We can set the number of iteration or can control the error rate between two iterations as the stopping condition. After several iterations, a satisfied segmented contour could be obtained, which is on or approaching the real edge because of the influence of maximum probability of the model, even if the part of real edge is lost. Thus, we can get a satisfied result.

After segmented one slice, the algorithm can be automatically performed to segment next slice until all slices of the selected object are done. The segmented slices can be constructed into a segmented volume.

Figure 3 shows four different images and the segmentation results. The method works well for both "good" and "bad" images and for images with discontinuous edges. The segmentation was assessed by the overlap ratio between automatic and manual segmentation and by mean distance errors between the two segmentations. For MR images from 24 mice, the average and standard deviation of the overlap ratios is $0.93 \pm 0.05$. The distance error between the automatic and manual segmentation is $0.85 \pm 0.41$ pixel. The method is accurate and robust for mouse kidney MR images.

\section{CONCLUSION}

We developed an automatic minimal path segmentation method for kidney MR images. In order to improve the robustness of the segmentation method, we incorporated distance and gradient information into the energy function to guide the marching curve toward the best path and span the broken edge. In this new method, the prior knowledge model was used to assist the potential curve to select the best path. During the dynamic programming search procedure, the placement and update of the end point were automatically performed in our new method. The automatic segmentation method is fast, accurate, and robust. This method provides a useful tool for quantification and characterization of polycystic kidney disease in transgenic mice. It can be applied to human kidney images with many clinical applications.

\section{ACKNOWLEDGMENT}

This work was partially supported by NIH grant R21CA120536 (PI: B Fei), by a Pilot Grant from the Case Comprehensive Cancer Center (PI: B Fei), and by Case Western Reserve University Presidential Research Initiative Award (PI: B Fei). The imaging facility is supported by the Northeastern Ohio Animal Imaging Resource Center as funded by NIH grant 5R24CA110943.

\section{REFERENCES}

[1] BW. Fei, C. Flask, and HS. Wang Image, "Segmentation, Registration and Visualization of Serial MR Images for Therapeutic Assessment of Polycystic Kidney Disease in Transgenic Mice," IEEE Engineering in Medicine and Biology 27th Annual Conference, pp. 467-469, 2005

[2] M. Kass, A. Witkin, and D. Terzopoulos, Snake: Active contour models. Int. J. Comput. Vis., Vol 1(4), pp. 321-331, 1987

[3] V. Caselles, F. Catte, T. Coll, and F. Dibos, "A geometric model for active contours," Numerische Mathematik, vol. 66, pp. 1-31, 1993.

[4] T. F. Cootes, A. Hill, C. J. Taylor, and J. Haslam, "Use of active shape models for locating structures in medical images," Imag. Vis. Computing J., vol. 12, no. 6, pp. 355-366, 1994.

[5] D. Geiger, A. Gupta, L. A. Costa, and J. Vlontzos, "Dynamic programming for detecting, tracking and matching deformable contours," IEEE Trans. Pattern Anal. Machine Intell., vol. 17, pp. 294302, Mar. 1995.

[6] PK Yan, and A. A. Kassim, Medical Image Segmentation Using Minimal Path Deformable Models With Implicit Shape Priors, IEEE Trans. Info. Tech. in Bio, Vol 10(4), pp. 677-684, 2006 